Journal of Fluid Mechanics

http://journals.cambridge.org/FLM

Additional services for Journal of Fluid Mechanics:

Journal of

Fluid Mechanics

Email alerts: $\underline{\text { Click here }}$

Subscriptions: $\underline{\text { Click here }}$

Commercial reprints: $\underline{\text { Click here }}$

Terms of use : $\underline{\text { Click here }}$

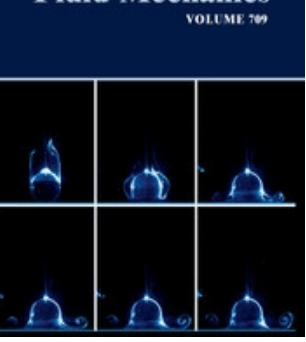

\title{
Singular modes in Rayleigh instability of three-dimensional streamwise- vortex flows
}

S. N. TIMOSHIN and F. T. SMITH

Journal of Fluid Mechanics / Volume 333 / February 1997, pp 139 - 160

DOI: 10.1017/S0022112096004181, Published online: 08 September 2000

Link to this article: http://journals.cambridge.org/abstract_S0022112096004181

How to cite this article:

S. N. TIMOSHIN and F. T. SMITH (1997). Singular modes in Rayleigh instability of three-dimensional streamwise-vortex flows. Journal of Fluid Mechanics, 333, pp 139-160 doi:10.1017/S0022112096004181

Request Permissions : $\underline{\text { Click here }}$ 


\title{
Singular modes in Rayleigh instability of three-dimensional streamwise-vortex flows
}

\author{
By S. N. TIMOSHIN AND F. T. SMITH \\ Department of Mathematics, University College London, Gower Street, \\ London WC1E 6BT, UK
}

(Received 4 May 1995 and in revised form 20 August 1996)

The upper-branch neutral modes of inviscid instability in a boundary-layer flow with significant longitudinal vortices present are shown to possess typically a logarithmically singular, non-inflectional, critical layer. This contrasts with previous linear and nonlinear suggestions implemented in vortex-wave interaction and secondary instability theories, which are re-examined. The analysis here is based first on perturbation techniques applied to a Rayleigh unstable planar motion supplemented by a vortex centred around the inflection level, followed by the extension to more general cases. Flows with order one and larger spanwise scales are considered. Multiple solutions, their limit properties and parametric continuations are illustrated with concrete examples.

\section{Introduction}

The inflection-point condition is extremely well known, going back to the nineteenth century study by Rayleigh (1880). It applies to the stability of locally quasiunidirectional basic flows for inviscid disturbances and it means, coupled with the Reynolds-stress integral, that neutral waves on the verge of instability travel with exactly the same (phase) speed as the main flow at the point of vanishing curvature in the velocity profile. Because of that, the wave motion can be described in terms of regular functions; see e.g. Lin (1955), Betchov \& Criminale (1967), or most other texts on hydrodynamic stability. It works for virtually any flow with a single point of inflection in the middle, as, for instance, in the conventional pressure-driven boundary layer in two dimensions. It does not work, however, for certain other realistic situations such as for basic flows with substantial cross-plane variations, e.g. longitudinal vortices, where the Reynolds-stress integration becomes affected significantly by the 'spanwise' dependence. Indeed, concrete examples found below (and, in a different setting, independently by Goldstein \& Wundrow 1995) indicate that in such cases the neutral modes are in general non-inflectional and hence singular. Our particular interest was triggered specifically by the findings in a theoretical paper on transition via nonlinear vortex-wave interaction (Brown et al. 1993) and a puzzling feature in the higher-order contributions, and this puzzle led back to the linear and nonlinear works by Hall \& Horseman (1991) and Hall \& Smith (1991), respectively, which are discussed/amended below. The resulting implications are believed to be much broader.

In this paper we study properties of an inviscid, upper-branch, neutral or temporally growing instability wave in a spanwise-periodic boundary layer with relatively strong longitudinal vortex structures. Among the first to consider this problem on a rational basis were the simultaneous papers by Hall \& Horseman (1991) and Hall \& Smith (1991), hereinafter referred to as HH and HS, respectively, although the topic of 
inviscid instabilities in a general uni-directional flow dates back to an early paper by Hocking (1968). The prime concern in HH was with linear computations, seeking the most unstable modes inherent in centrifugally driven vortex rolls on concave surfaces; for a related work on heated plates see Hall (1993). Although the numerical technique used in $\mathrm{HH}$ failed to produce solutions close to the neutral point, a fair amount of evidence was given, nevertheless, to support firstly the inflectional nature of the dominant instabilities (in the sense that profiles without a point of inflection tend to be stable) and secondly non-uniqueness (i.e. multiplicity) of the unstable modes related to the spanwise periodicity of the vortex boundary layer. Other related linear studies likewise tending to concentrate away from the neutral state include those of Henningson (1987), Balachandar, Streett \& Malik (1992), Malik, Li \& Chang (1994) and Otto (1996). The study in HS addresses the problem in the context of nonlinear interactions between inviscid instabilities and induced or input streamwise vortices. There it was suggested that interaction may reach a saturated stage in which the wavedriven vortex field maintains the wave system in a neutral state at each streamwise location in the flow (cf. Smith, Brown \& Brown 1993). Properties of the neutral modes are, therefore, of major significance for the vortex-wave interaction theory and that, in fact, was where our interest in the subject arose in the first place. In HS (and Blackaby \& Hall 1995) it was assumed, by analogy with the Rayleigh inflectional criterion in two dimensions, that the neutral waves in a vortex flow must be regular at a critical layer where the waves' phase speed coincides with the velocity of the main flow. Subsequently, however, a local analysis in Brown et al. (1993) of the HS-type flow induced by a pair of oblique waves impinging onto a Rayleigh-unstable planar boundary layer revealed a rather subtle controversy (Brown et al. §9) in the vortex-wave interaction theory stemming from the assumed regularity of the neutral wave motion.

The aim in the present work is to describe a theoretical analysis of near-neutral instabilities in a model formulation appropriate to both the secondary instability and the vortex-wave interaction settings above. In consequence, we shall be working with a basic vortex flow which, to start with, is assumed to have its typical cross-scales comparable with the main boundary-layer thickness, as in HH and HS. The derivation of the stability equation for such flows and the issue of solvability of the vortex-wave interaction equations raised in Brown et al. (1993) are summarized below in this section. In order to shed light analytically on the computational difficulties encountered in $\mathrm{HH}$, the main-flow velocity field is subsequently treated as a superposition of an inflectional planar component and three-dimensional vortex components centred around the inflection point, after which more general cases are considered. The overall structure of the basic flow is similar to the starting HS-type vortex considered in Brown et al. (1993), although the analysis here is carried out somewhat more in the vein of the $\mathrm{HH}$ approach. The characteristic amplitude of the vortex is initially taken to be small, so that marginally unstable three-dimensional modes are obtained as perturbations about the conventional, neutral and regular, planar Rayleigh solution, before the more general case is considered, as described in $\S 2$. The simplifications achieved turn out to be so considerable that the eigenvalue formulation for the growth rate of instability, for example, reduces to a transcendental algebraic equation. This part of the theory has some similarities to the treatment in Goldstein \& Wundrow (1995) of inviscid instabilities in a flat-plate Blasius boundary layer weakly distorted by a vortex (which we note is assumed to be artificially generated in a near-wall part of the flow), although our scalings and the algebraic detail are somewhat different. Also, as in that paper, the primary result given in $\S 2$ concerns the smoothness of the wave solutions with 

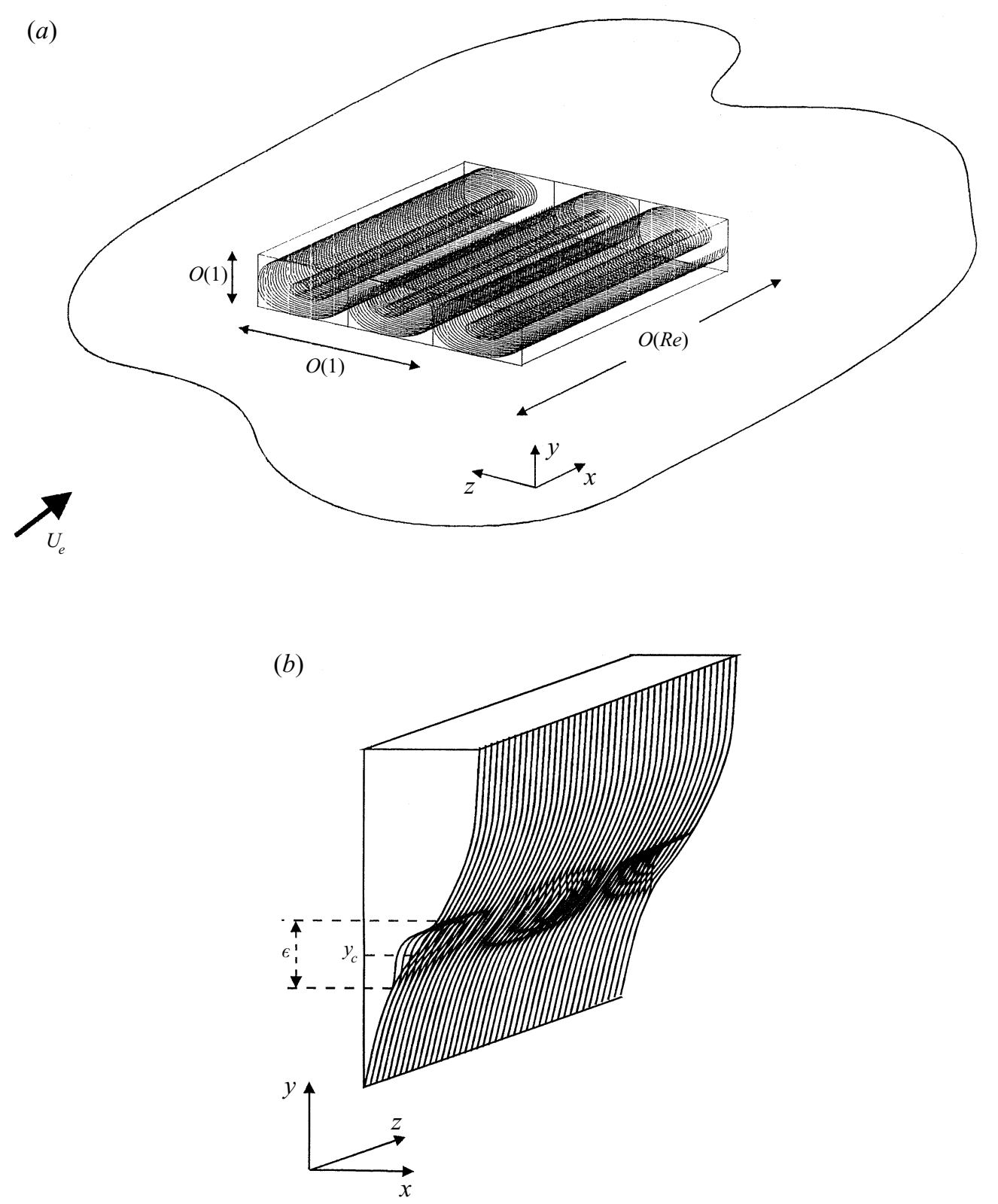

FIGURE 1. (a) The sketch illustrates the typical scales of a longitudinal vortex flow implied in the derivation of the inviscid instability equation (1.4). (b) The streamwise velocity profiles shown qualitatively for the case of a weak vortex concentrated near the inflection point $y_{c}$ in the basic planar boundary layer, the scaled thickness of the vortex $\epsilon$ serving as the small parameter in the perturbation analysis in $\S \S 2$ and 3 .

vanishing growth rates. It is shown, by means of a direct solution of the perturbation equations, that in contrast with the two-dimensional Rayleigh theory the neutral modes in a vortex boundary layer contain a logarithmic singularity and a 'phase-shift' discontinuity at the critical level. In our analysis, this follows from a purely inviscid argument without need to resolve a viscous critical-layer structure as in Goldstein \& Wundrow (1995). In order to capture the second main effect, namely non-uniqueness 
of a spanwise periodic wave, as observed in the computations of $\mathrm{HH}$, the stability of the flow with a somewhat increased cross-scale is studied in $\S 3$. Following the perturbation procedure of $\S 2$, the problem reduces to an eigenvalue formulation for a second-order differential equation with periodic coefficients, which is analysed with regard to certain additional limiting cases. Three specific examples are considered briefly in $\S 4$. A discussion of the results concerning their application to vortex-wave interactions and to secondary instability theories is given in conclusion (§5). The main results overall, however, are the two italicized above, namely the non-inflectional logarithmically singular critical layer and the non-uniqueness.

\subsection{The pressure disturbance equation}

In the derivation of the controlling equation for instability of the vortex flow we assume, following $\mathrm{HH}$ and $\mathrm{HS}$, that the longitudinal vortex axis is aligned with the predominantly two-dimensional free stream at the outer edge of the boundary layer and the cross-scale of the vortex is comparable with the boundary-layer thickness (see figure $1 a$ ). Then the main flow in the boundary layer is also along the free stream, whereas the transverse vortex motion is much weaker and, consequently, has no impact on the stability properties. The fluid is assumed to be incompressible, for simplicity.

Suppose, next, that all quantities in the equations of motion have been nondimensionalized using the boundary-layer thickness at a chosen location as the typical lengthscale and the velocity at the outer edge of the boundary layer $U_{e}$ as the reference speed. The variable part of the pressure is referred to $\rho U_{e}^{2}, \rho$ being the density, and the Reynolds number is denoted as $R e$. In such variables the mean streamwise velocity in the boundary layer $u$ turns out to be a function of the spanwise and normal coordinates, $z$ and $y$, respectively, slowly varying on the relatively large development length of order Re along the free stream. We denote $x$ to be the fast streamwise variable, $x=O(1)$ on the scale of the boundary-layer thickness, and $X=R e^{-1} x$ to be the slow-scale coordinate for the vortex-flow development. The cross-flow velocities in the vortex, $v$ and $w$ along $y$ and $z$, respectively, are estimated as $O\left(R e^{-1}\right)$, e.g. from mass conservation. The wave disturbances, on the other hand, are (conventionally) assumed to have all three spatial scales of order unity, so that the streamwise dependence in the main flow can be neglected in the leading approximation of the stability analysis. Also the solid boundary can be regarded as a flat plate at $y=0$ locally. Hence, the perturbed-flow components are written in the form

$$
(u, v, w, p)=\left(u_{0}(X, y, z), 0,0, p_{0}\right)+\delta\{E(\bar{u}, \bar{v}, \bar{w}, \bar{p})+\text { c.c. }\}+O\left(\delta^{2}\right),
$$

where $u_{0}(X, y, z)$ is the main flow velocity, $p_{0}$ is a constant and $\delta \ll 1$ measures the typical amplitude of disturbances; the notation c.c. is used for the complex conjugate. The wave behaviour of the disturbance has been incorporated in the factor $E=\exp [\mathrm{i} \alpha(x-c t)]$, with $t$ being non-dimensional time and $\alpha$ and $c$ the wavenumber and phase speed, respectively. The barred disturbance components are functions of slow $X$ and of the transverse coordinates governed, on substituting (1.1) into the Navier-Stokes equations and neglecting higher-order nonlinear, non-parallel and viscous terms, by the system of inviscid equations

$$
\begin{gathered}
\mathrm{i} \alpha\left(u_{0}-c\right) \bar{u}+\bar{v} \frac{\partial u_{0}}{\partial y}+\mathrm{i} \alpha \bar{p}=0, \quad \mathrm{i} \alpha\left(u_{0}-c\right) \bar{v}+\frac{\partial \bar{p}}{\partial y}=0, \\
\mathrm{i} \alpha\left(u_{0}-c\right) \bar{w}+\frac{\partial \bar{p}}{\partial z}=0, \quad \mathrm{i} \alpha \bar{u}+\frac{\partial \bar{v}}{\partial y}+\frac{\partial \bar{w}}{\partial z}=0,
\end{gathered}
$$


from the momentum balances in the $x$-, $y$-, $z$-directions and from continuity, respectively. The perturbed velocities can be eliminated from the system to obtain the pressure disturbance equation

$$
\left(u_{0}-c\right)\left(\frac{\partial^{2} \bar{p}}{\partial y^{2}}+\frac{\partial^{2} \bar{p}}{\partial z^{2}}-\alpha^{2} \bar{p}\right)=2\left(\frac{\partial u_{0}}{\partial y} \frac{\partial \bar{p}}{\partial y}+\frac{\partial u_{0}}{\partial z} \frac{\partial \bar{p}}{\partial z}\right),
$$

as in Hocking (1968), HH, HS and related studies. The boundary conditions on the perturbed pressure function are

$$
\partial \bar{p} / \partial y=0 \quad \text { at } \quad y=0, \quad \bar{p} \rightarrow 0 \quad \text { as } \quad y \rightarrow \infty,
$$

from no penetration at the surface and the decay at infinity, respectively.

In the context of linear secondary instability $u_{0}$ is treated as a given function of $y$ and $z$ with the slow variable $X$ playing the role of a parameter. One main interest is then in the fastest growing modes, but for certain purposes (as e.g. in Blackaby \& Hall (1995) analysing weakly-nonlinear modulation of the secondary modes) neutrally stable solutions of (1.4) and (1.5) may be necessary. The problem is then similar, to a certain extent, to that in the HS theory where the wave motion is supposed to be neutral at each $X$ for the link between the mean velocity $u_{0}$ and the wave pressure $\bar{p}$ to be achieved via a system of Reynolds-stress effects. In general the vortex and the wave remain strongly coupled in this second formulation. Brown et al. (1993) demonstrate, however, that at the initial stages of interaction, close to the point of linear neutral stability $X=X_{n}$ for a pair of oblique waves with equal phase speeds and crosswavenumbers opposite in sign, the developing vortex is weak and hence can be described as a small perturbation to the basic-state planar profile,

$$
u_{0}=U_{0}(y)+\left(X-X_{n}\right)^{2} U_{1}(\eta, z)+\left(X-X_{n}\right)^{5 / 2} U_{2}(\eta, z) \ldots
$$

Here $\eta=\left(y-y_{c}\right) /\left(X-X_{n}\right)^{1 / 2}$, and $U_{1}, U_{2}$ include both non-parallel two-dimensional and wave-induced three-dimensional corrections. The wave pressure $\bar{p}$ also expands in power-series form at small $X-X_{n}$, with the leading-order solution being simply the neutral mode for the planar profile $U_{0}(y)$. Then the interaction conditions determine the starting wave amplitude $\bar{p}\left(X=X_{n}\right)$ to be a definite constant. The estimate for the required initial level of the wave disturbance is also confirmed in the shorter-scale theory in Smith et al. (1993). The analysis in Brown et al. (1993) indicates further that an attempt to improve the wave solution by including the vortex correction of the mean-flow distribution in (1.6) leads to a constraint on the analytic properties of the profile $U_{0}(y)$ at the critical point $y=y_{c}$ where $U_{0}^{\prime \prime}\left(y_{c}\right)$ is zero. Specifically, the solution of (1.4), (1.5) with (1.6) was found to exist provided that

$$
\frac{U_{0}^{(\mathrm{iv})}\left(y_{c}\right)}{U_{0}^{\prime \prime}\left(y_{c}\right)}=\frac{5}{2} \frac{U_{0}^{\prime}\left(y_{c}\right)}{U_{0}\left(y_{c}\right)},
$$

cf. (9.3) in Brown et al. (1993). The condition (1.7) is actually a consequence of the regularity assumed for the wave pressure at the critical level as clarified in the discussion around (9.1) in the last paper.

It seems bizarre here for solvability in a higher order to have such a strong impact on the overall existence of the flow at hand, especially when the most significant physics is incorporated in the main-order solution, which, to repeat, was supported in the independent analysis of a non-saturated interaction in Smith et al. (1993). In the next section, we argue that the difficulty stems from the critical-layer regularity condition 
which, as was also shown for the case of near-wall vortices in Goldstein \& Wundrow (1995), overspecifies the neutral-mode formulation.

\section{Singular neutral modes}

Solutions of (1.2) and (1.3) corresponding to unstable time-growing waves are smooth functions of the normal $y$-coordinate in the general case, on account of a strictly positive imaginary part of the phase speed, $c_{i}>0$. For a neutral disturbance, however, both $\alpha$ and $c$ are real; therefore the velocity components $\bar{u}, \bar{w}$ contain a pole singularity at the critical surface defined by the relation $u_{0}(y, z)=c$. This singularity is passive in the sense that it is smoothed out by viscous forces in a narrow inner layer without any effect on the solution of the inviscid equations (1.2)-(1.4); see e.g. Brown et al. (1993). Our concern is with a logarithmic singularity which, in principle, can appear in the third derivative of the pressure function $\partial^{3} \bar{p} / \partial y^{3}$ or equivalently in the velocity terms $\partial \bar{v} / \partial y$ and $\bar{u}$; for, if such a singularity does develop in the solution, an additional jump condition at the critical layer must be incorporated into the neutralwave formulation. In the case of a $z$-independent boundary layer the issue is resolved in the classical inviscid instability theory: in a flow with a single critical layer the singularity vanishes and consequently the critical layer must coincide with an inflection point in the main-flow velocity profile (see e.g. Lin 1955; Betchov \& Criminale 1967). Assuming the neutral solution of the Rayleigh problem for a two-dimensional profile $u_{0}=U_{0}(y)$ to be known, we shall first examine the regularity of the neutral modes and properties of the adjacent instabilities in a vortex flow with weak three-dimensional components concentrated near the inflection point in $U_{0}$; see figure $1(b)$. (This is generalized later in the present section.) In order to simplify the calculation a little, we shall take a somewhat higher level of the vortex disturbance than in (1.6), namely, suppose that

$$
u_{0}(y, z)=U_{0}(y)+\epsilon^{3} U_{1}\left(\frac{y-y_{c}}{\epsilon}, z\right),
$$

where $U_{1}$ is periodic in $z, \epsilon$ is a small parameter, and $U_{0}^{\prime \prime}\left(y_{c}\right)=0$. The solution of (1.4), (1.5) with (2.1) is sought as a perturbation to the planar neutral wave. Consider first the bulk of the flow where $y-y_{c}$ is $O(1)$ and expand the wave characteristics in powers of $\epsilon$ :

$$
\bar{p}=\bar{p}_{0}(y)+\epsilon \bar{p}_{1}(y, z)+\ldots, \quad \alpha=\alpha_{0}+\epsilon \alpha_{1}+\epsilon^{2} \alpha_{2}+\ldots, \quad c=c_{0}+\epsilon c_{1}+\epsilon^{2} c_{2}+\ldots,
$$

where $c_{0}, \alpha_{0}$ are real but $c_{1}=c_{1 r}+\mathrm{i} c_{1 i}$ is in general complex. The leading-order pressure disturbance $\bar{p}_{0}(y)$ represents the regular neutral-wave solution of the equation

$$
\left(U_{0}-c_{0}\right)\left(\bar{p}_{0}^{\prime \prime}-\alpha_{0}^{2} \bar{p}_{0}\right)=2 U_{0}^{\prime} \bar{p}_{0}^{\prime}, \quad c_{0}=U_{0}\left(y_{c}\right),
$$

whereas the correction term is governed by the inhomogeneous equation

$$
\left(U_{0}-c_{0}\right)\left(\frac{\partial^{2} \bar{p}_{1}}{\partial y^{2}}+\frac{\partial^{2} \bar{p}_{1}}{\partial z^{2}}-\alpha_{0}^{2} \bar{p}_{1}\right)-2 U_{0}^{\prime} \frac{\partial \bar{p}_{1}}{\partial y}=2 \alpha_{0} \alpha_{1}\left(U_{0}-c_{0}\right) \bar{p}_{0}+c_{1}\left(\bar{p}_{0}^{\prime \prime}-\alpha_{0}^{2} \bar{p}_{0}\right) .
$$

Both $\bar{p}_{0}$ and $\bar{p}_{1}$ must satisfy the boundary conditions (1.5).

In addition to the group of terms on the right-hand side of equation (2.4), the outer pressure $\bar{p}_{1}$ is forced by the vortex in the inner region where $Y=\left(y-y_{c}\right) \epsilon^{-1}$ is $O(1)$. When $y-y_{c}$ is small, the main-flow velocity and the leading pressure disturbance expand as

$$
U_{0}=c_{0}+b_{1}\left(y-y_{c}\right)+\frac{1}{6} b_{3}\left(y-y_{c}\right)^{3}+\ldots, \quad \bar{p}_{0}=p_{00}+\frac{1}{2} p_{02}\left(y-y_{c}\right)^{2}+\frac{1}{6} p_{03}\left(y-y_{c}\right)^{3}+\ldots,
$$


with constant coefficients $b_{j}, p_{0 j}$. The expansion for the wave pressure in the inner zone therefore proceeds as

$$
\bar{p}=P_{0}+\epsilon P_{1}+\epsilon^{2} P_{2}+\epsilon^{3} P_{3}+\epsilon^{4} P_{4}+\ldots,
$$

where, on substituting into (1.4) and matching with the outer solution,

$$
\begin{gathered}
P_{0}=p_{00}, \quad P_{1}=P_{1}(z)=\bar{p}_{1}\left(y_{c}, z\right), \quad P_{2}=P_{20}(z)-\frac{\alpha_{0}^{2} p_{00}}{2 b_{1}^{2}}\left(b_{1} Y-c_{1}\right)^{2}, \\
P_{3}=P_{30}(z)+\frac{c_{2} \alpha_{0}^{2} p_{00}}{b_{1}^{2}}\left(b_{1} Y-c_{1}\right)-\frac{1}{2 b_{1}^{2}}\left(\alpha_{0}^{2} P_{1}-P_{1}^{\prime \prime}+2 \alpha_{0} \alpha_{1} p_{00}\right)\left(b_{1} Y-c_{1}\right)^{2} \\
+\frac{p_{03}}{6 b_{1}^{3}}\left(b_{1} Y-c_{1}\right)^{3} .
\end{gathered}
$$

The specific form of the functions $P_{1}, P_{20}, P_{30}$ in the solution above is insignificant for our purposes. The crucial formulation containing the first appearance of the vortex effect in the inner layer arises at order $\epsilon^{4}$. The pressure term $P_{4}$ in (2.6) consists of a purely polynomial part which, similarly to the earlier terms, matches with the outer solution automatically, and a vortex-induced contribution governed by the equation

$$
\left(b_{1} Y-c_{1}\right) \frac{\partial^{2} P_{4}}{\partial Y^{2}}-2 b_{1} \frac{\partial P_{4}}{\partial Y}=-2 \alpha_{0}^{2} p_{00}\left\{\left(\frac{1}{2} b_{3} Y^{2}+\frac{\partial U_{1}}{\partial Y}\right)\left(Y-\frac{c_{1}}{b_{1}}\right)-\left(\frac{1}{6} b_{3} Y^{3}+U_{1}\right)\right\} .
$$

Here, on the right-hand side, we have retained the main curvature term of the basicflow profile in (2.5). Considering first the case of unstable time-growing disturbances with $c_{1 i}>0$, we integrate (2.9) to obtain

$$
\frac{\partial P_{4}}{\partial Y}=-\frac{\alpha_{0}^{2} p_{00}}{b_{1}}\left\{U_{*}-\left(Y-\frac{c_{1}}{b_{1}}\right) \frac{\partial U_{*}}{\partial Y}+\left(Y-\frac{c_{1}}{b_{1}}\right)^{2}\left[P_{43}(z)+\int_{Y_{0}}^{Y} \frac{\partial^{2} U_{*}(s, z)}{\partial s^{2}} \frac{\mathrm{d} s}{s-c_{1} b_{1}^{-1}}\right]\right\}
$$

where $U_{*}(Y, z)=\frac{1}{6} b_{3} Y^{3}+U_{1}(Y, z)$ represents the 'effective' vortex velocity; the function $P_{43}(z)$ and the lower limit of integration $Y_{0}$ are arbitrary. Hence, the behaviour of the solution at the outer edges of the inner region is found to be of the form

$$
P_{4}=-\frac{\alpha_{0}^{2} p_{00}}{b_{1}}\left[\frac{b_{3} c_{1}}{3 b_{1}} Y^{3} \ln |Y|+A^{ \pm}(z) Y^{3}+\ldots\right],
$$

as $Y \rightarrow \pm \infty$ in accordance with the superscript to $A^{ \pm}$. The jump in the coefficient of the cubic term is then evaluated as

$$
A^{+}-A^{-}=\frac{1}{3} \int_{-\infty}^{\infty} K_{1}(Y, z) \frac{\mathrm{d} Y}{Y-c_{1} b_{1}^{-1}}+\frac{\mathrm{i} \pi b_{3} c_{1}}{3 b_{1}}
$$

assuming that the curvature of the vortex velocity $K_{1}(Y, z) \equiv \partial^{2} U_{1} / \partial Y^{2}$ decays sufficiently fast for convergence of the integral. In order to match the logarithmic term in (2.11) with the outer solution, an additional contribution of the form $Y^{3} \ln \epsilon$ times (a function of $z$ ) must be included in $P_{4}$ in the usual manner. Then (2.11), together with 
the matching conditions for the previous approximations in (2.6) and (2.8) indicate that the outer disturbance pressure $\bar{p}_{1}$, as well as its first and second $y$-derivatives, must be continuous at $y_{c}$, whereas the third derivative behaves according to

$$
\frac{\partial^{3} \bar{p}_{1}}{\partial y^{3}}=-\frac{2 \alpha_{0}^{2} p_{00} b_{3} c_{1}}{b_{1}^{2}}\left[\ln \left|y-y_{c}\right|+\frac{3 b_{1}}{b_{3} c_{1}} A^{ \pm}(z)+\frac{11}{6}+o(1)\right],
$$

as $y \rightarrow y_{c} \pm 0$ on approach to the inner region.

We show next how the solution of (2.4) with the trivial conditions at the wall and at infinity (1.5), and with the jump condition (2.13) and (2.12), provides a dispersion relation for the phase speed $c_{1}$ in terms of the wavenumber variation $\alpha_{1}$. The spanwiseperiodic vortex velocity $U_{1}$ and hence the curvature $K_{1}$ expand in infinite Fourier series in $z$ in the general case. However, for the purpose of illustration, we can confine ourselves to the two-term representations

$$
\begin{gathered}
U_{1}=U_{m}(Y)+U_{\beta}(Y) \cos \beta z, \quad K_{1}=K_{m}(Y)+K_{\beta}(Y) \cos \beta z, \\
\bar{p}_{1}=p_{m}(y)+p_{\beta}(y) \cos \beta z,
\end{gathered}
$$

for the vortex velocity and curvature in the inner region, and the wave pressure in the bulk, respectively. On substituting the last of the above relations into (2.4) we find that the mean component of the disturbance pressure $p_{m}$ is governed by the following formulation,

$$
\begin{gathered}
\left(U_{0}-c_{0}\right)\left(p_{m}^{\prime \prime}-\alpha_{0}^{2} p_{m}\right)-2 U_{0}^{\prime} p_{m}^{\prime}=2 \alpha_{0} \alpha_{1}\left(U_{0}-c_{0}\right) \bar{p}_{0}+c_{1}\left(\bar{p}_{0}^{\prime \prime}-\alpha_{0}^{2} \bar{p}_{0}\right) ; \quad p_{m}^{\prime}(0)=p_{m}(\infty)= \\
p_{m}^{\prime \prime \prime}=-\frac{2 \alpha_{0}^{2} p_{00} b_{3} c_{1}}{b_{1}^{2}}\left[\ln \left|y-y_{c}\right|+\frac{3 b_{1}}{b_{3} c_{1}} A_{m}^{ \pm}+\frac{11}{6}+o(1)\right] \quad \text { as } \quad y \rightarrow y_{c} \pm 0,
\end{gathered}
$$

on account of (1.5) and (2.13), with

$$
A_{m}^{+}-A_{m}^{-}=\frac{1}{3} \int_{-\infty}^{\infty} K_{m}(Y) \frac{\mathrm{d} Y}{Y-c_{1} b_{1}^{-1}}+\frac{\mathrm{i} \pi b_{3} c_{1}}{3 b_{1}}
$$

giving the $z$-averaged part of the jump (2.12). Provided that the eigenmode $\bar{p}_{0}$ is known, the solution of the inhomogeneous Rayleigh equation (2.15) can be found explicitly (see e.g. Smith et al. 1993). Setting $p_{m}=\bar{p}_{0} Q(y)$ we obtain

$$
\frac{Q^{\prime} \bar{p}_{0}^{2}}{\left(U_{0}-c_{0}\right)^{2}}=\int_{y_{b}}^{y}\left[2 \alpha_{0} \alpha_{1}\left(U_{0}-c_{0}\right) \bar{p}_{0}+c_{1}\left(\bar{p}_{0}^{\prime \prime}-\alpha_{0}^{2} \bar{p}_{0}\right)\right] \frac{\bar{p}_{0} \mathrm{~d} y}{\left(U_{0}-c_{0}\right)^{3}}
$$

where the limit of integration $y_{b}=0$ if $y<y_{c}$, and $y_{b}=\infty$ if $y>y_{c}$. Hence the pressure function $p_{m}$ is non-analytic at $y_{c}$, and, given the continuity up to the second derivative, for the third derivative near $y_{c}$ we have the relation

$$
p_{m}^{\prime \prime \prime}=-\frac{2 \alpha_{0}^{2} p_{00} b_{3} c_{1}}{b_{1}^{2}} \ln \left|y-y_{c}\right|+R^{ \pm}+o(1) \quad \text { as } \quad y \rightarrow y_{c} \pm 0,
$$

where

$$
R^{+}-R^{-}=-\frac{2 b_{1}^{2}}{p_{00}}\left[c_{1} q_{1}+2 \alpha_{0} \alpha_{1} q_{2}\right],
$$


and the real constants $q_{1,2}$ are defined as the finite parts of the integrals

$$
q_{1}=\int_{0}^{\infty} \frac{\bar{p}_{0}\left(\bar{p}_{0}^{\prime \prime}-\alpha_{0}^{2} \bar{p}_{0}\right)}{\left(U_{0}-c_{0}\right)^{3}} \mathrm{~d} y, \quad q_{2}=\int_{0}^{\infty} \frac{\bar{p}_{0}^{2} \mathrm{~d} y}{\left(U_{0}-c_{0}\right)^{2}} .
$$

Matching (2.19) and (2.20) with the inner-layer jump condition in (2.16) and (2.17) yields the required dispersion relation

$$
c_{1} q_{1}+2 \alpha_{0} \alpha_{1} q_{2}=\frac{\alpha_{0}^{2} p_{00}^{2}}{b_{1}^{3}}\left[\int_{-\infty}^{\infty} K_{m}(Y) \frac{\mathrm{d} Y}{Y-c_{1} b_{1}^{-1}}+\frac{\mathrm{i} \pi b_{3} c_{1}}{b_{1}}\right],
$$

controlling $c_{1}$ for given $\alpha_{1}$, say. An alternative derivation of (2.22) can be made using the technique of adjoint solutions.

It is important to note that the complex phase speed $c_{1}=c_{1}\left(\alpha_{1}\right)$ determined in principle by (2.22) depends entirely on the $z$-average component of the vortex flow. If, for instance, the mean curvature $K_{m} \equiv 0$, then instability can only be due to variations in the wavenumber $\alpha_{1}$. A neutral wave is obtained then only if $c_{1}=0$ and then also $\alpha_{1}=0$, taking successively the imaginary and real parts of (2.22), so that the main planar wave remains unaltered in the current approximation. Otherwise the mean vortex curvature $K_{m}$ alters the growth rate of the disturbance, for non-zero $U_{m}$. For the neutral wave in this case the imaginary part of (2.22) evaluated in the limit $c_{1 i} \rightarrow 0+$ shows that the critical level $Y=c_{1} / b_{1}$ coincides with the inflection point in the total $z$ averaged velocity $U_{m}(Y)+\frac{1}{6} b_{3} Y^{3}$. This in fact determines the main correction to the location of the critical layer, $y=y_{c}+\epsilon c_{1} b_{1}^{-1}+O\left(\epsilon^{2}\right)$. All such conclusions follow immediately from the inflection-point condition applied to a slightly altered planar main flow. A non-trivial result is obtained, however, from examination of the regularity properties of the neutral wave disturbance in the inner region. It follows from (2.10) that the pressure term $P_{4}$ turns out to be singular at $Y=c_{1} b_{1}^{-1}$ in the neutral-wave limit, $c_{1 i} \rightarrow 0$, for any flow with a non-vanishing $\beta$-harmonic in its velocity field in (2.14). More specifically we have

$$
P_{4}=\ldots-\frac{\alpha_{0}^{2} p_{00}}{3 b_{1}} \frac{\partial^{2} U_{*}}{\partial Y^{2}}\left(Y=\frac{c_{1}}{b_{1}}, z\right)\left(Y-\frac{c_{1}}{b_{1}}\right)^{3} \ln \left|Y-\frac{c_{1}}{b_{1}}\right|+\ldots
$$

as $Y \rightarrow c_{1} b_{1}^{-1}$, omitting the regular part preceding the first singular term shown explicitly. In addition to the logarithmic singularity, the next term in the expansion (2.23) contains a non-zero critical-layer jump evaluated as

$$
P_{4}\left(\frac{c_{1}}{b_{1}}+0, z\right)-P_{4}\left(\frac{c_{1}}{b_{1}}-0, z\right)=-\mathrm{i} \pi \frac{\alpha_{0}^{2} p_{00}}{3 b_{1}} \frac{\partial^{2} U_{*}}{\partial Y^{2}}\left(Y=\frac{c_{1}}{b_{1}}, z\right)\left(Y-\frac{c_{1}}{b_{1}}\right)^{3}+\ldots
$$

Having established the wave parameters by means of (2.22) we can now verify solvability for the harmonic $p_{\beta}$ in (2.14). The controlling equations and conditions are of the form

$$
\begin{aligned}
& \left(U_{0}-c_{0}\right)\left(p_{\beta}^{\prime \prime}-\left(\alpha_{0}^{2}+\beta^{2}\right) p_{\beta}\right)-2 U_{0}^{\prime} p_{\beta}^{\prime}=0, \quad p_{\beta}^{\prime}(0)=p_{\beta}(\infty)=0, \\
& p_{\beta}^{\prime \prime \prime}\left(y_{c}+0\right)-p_{\beta}^{\prime \prime \prime}\left(y_{c}-0\right)=J=-\frac{2 \alpha_{0}^{2} p_{00}}{b_{1}} \int_{-\infty}^{\infty} U_{\beta}^{\prime \prime}(Y) \frac{\mathrm{d} Y}{Y-c_{1} b_{1}^{-1}},
\end{aligned}
$$

as follows from (2.4), (2.12)-(2.14) and (1.5). Since the total wavenumber $\left(\alpha_{0}^{2}+\beta^{2}\right)^{1 / 2}$ differs from the eigenvalue $\alpha_{0}$ corresponding to the phase speed $c_{0}$, the solution of 
(2.25) is uniquely determined by the jump value $J$ in (2.26). This can be shown using a Frobenius argument in the vicinity of $y_{c}$. Alternatively, explicit solutions can be obtained when $\beta \rightarrow 0$ or $\beta \rightarrow \infty$. In the latter case, for example, the three-dimensional part of the wave disturbance is concentrated in a layer of thickness $O\left(\beta^{-1}\right)$ near $y_{c}$, the corresponding limit solution being explicitly

$$
p_{\beta}=\frac{1}{4 \beta^{3}} J\left[1 \pm \beta\left(y-y_{c}\right)\right] \exp \left[\mp \beta\left(y-y_{c}\right)\right]+o\left(\frac{1}{\beta^{3}}\right), \quad \beta \gg 1, \quad y \gtrless y_{c} .
$$

The former case of the limit $\beta \rightarrow 0$ is of much interest and will be considered in $\S 3$ from a more general viewpoint.

The relation (2.10) illustrates similarities (and differences) between the flow regimes considered here and in Goldstein \& Wundrow (1995). Their vortex develops in a nearwall part of the planar Blasius flow with the local property $U_{0}=\lambda_{0} y+\lambda_{1} y^{4}+\ldots$ as $y \rightarrow 0$ (in our notation). Vortex corrections can well destabilize the flow on their own; however, a characteristic competition with the 'natural' curvature of the Blasius profile is achieved when the vortex intensity is of order $y^{4}$ at a chosen small $y$. In terms of our (2.10) this means the inner-flow profile of the form $U_{*}=\lambda_{1} Y^{4}+U_{1}$, instead of the formula given just after (2.10), with the lower limit in the integrals (2.12) and onwards replaced by zero. The form of the $\epsilon$-expansions in the analysis above is also different.

\subsection{The singularity in the full stability formulation}

A Frobenius-type argument applied to the case of neutral stability of a strong $O(1)$ vortex indicates that (1.4) allows both regular (HS; Blackaby \& Hall 1995) and singular (Wundrow \& Goldstein 1994) wave-pressure distribution near the critical layer locally. It is instructive to compare the two options from the viewpoint of the solvability problem encountered, in particular, in Brown et al. (1993). In the neighbourhood of the critical surface $y=f(z)$ such that $u_{0}(f(z), z)=c$, the mean velocity and the pressure function expand in the form

$$
\begin{gathered}
u_{0}(y, z)=c+(y-f) \hat{u}_{1}(z)+\frac{1}{2}(y-f)^{2} \hat{u}_{2}(z)+\frac{1}{6}(y-f)^{3} \hat{u}_{3}(z)+\ldots, \\
\bar{p}(y, z)=\hat{p}_{0}(z)+(y-f) \hat{p}_{1}(z)+\frac{1}{2}(y-f)^{2} \hat{p}_{2}(z)+\frac{1}{6}(y-f)^{3}\left[\hat{p}_{30}(z) \ln |y-f|+\hat{p}_{31}^{ \pm}(z)\right]+\ldots,
\end{gathered}
$$

respectively. On substituting into (1.4), the coefficients in (2.29) are linked by means of the differential relations

$$
\begin{gathered}
\hat{p}_{1}\left(1+f^{\prime 2}\right)=\hat{p}_{0}^{\prime} f^{\prime} \\
\hat{p}_{2}\left(1+f^{\prime 2}\right)=\hat{p}_{0}^{\prime \prime}-\alpha^{2} \hat{p}_{0}-f^{\prime \prime} \hat{p}_{1}+2 \hat{u}_{1}^{\prime}\left(f^{\prime} \hat{p}_{1}-\hat{p}_{0}^{\prime}\right) / \hat{u}_{1} \\
\hat{p}_{30}=-\frac{2}{\hat{u}_{1}}\left[2 \hat{u}_{1}^{\prime}\left(f^{\prime} \hat{p}_{2}-\hat{p}_{1}^{\prime}\right)+\hat{u}_{2}^{\prime}\left(f^{\prime} \hat{p}_{1}-\hat{p}_{0}^{\prime}\right)+\frac{1}{2} \hat{u}_{2}\left(2 f^{\prime} \hat{p}_{1}^{\prime}-3\left(1+f^{\prime 2}\right) \hat{p}_{2}+\hat{p}_{0}^{\prime \prime}-\alpha^{2} \hat{p}_{0}-f^{\prime \prime} \hat{p}_{1}\right)\right. \\
\left.+\hat{u}_{1}\left(\hat{p}_{1}^{\prime \prime}-\alpha^{2} \hat{p}_{1}-\left(f^{\prime} \hat{p}_{2}\right)^{\prime}\right)\right]
\end{gathered}
$$

Also for the discontinuity in the cubic term in (2.29) we have the quasi-twodimensional result

$$
\hat{p}_{31}^{+}-\hat{p}_{31}^{-}=\mathrm{i} \pi \hat{p}_{30}
$$

as follows, for example, from analysis of near-neutral modes with small positive growth rates.

For the regular mode with $\hat{p}_{30}(z) \equiv 0$ the relations (2.30)-(2.32) turn into a system of equations for the three unknown functions $\hat{p}_{0}, \hat{p}_{1}, \hat{p}_{2}$ in terms of the critical-layer 
shape $f$ and the coefficients in (2.28). If, however, a singular behaviour is permitted in (2.29), then the system acquires an additional freedom through the unknown $\hat{p}_{30}$ or, equivalently, through the jump $\hat{p}_{31}^{+}-\hat{p}_{31}^{-}$in (2.33). In a sense, the second singular choice appears to be less restrictive locally and therefore is more likely to occur in the complete solution, as is indeed observed above and in Goldstein \& Wundrow (1995).

Neither our analysis nor the model flow examined in Goldstein \& Wundrow (1995) deny regular neutral solutions in some exceptional cases. Nevertheless the bulk of the available asymptotic results for weak vortices points to the singular behaviour as the most typical outcome for a general strong vortex field.

\section{Non-uniqueness of the neutral modes}

The perturbation procedure of the previous section completely ignores the selectivity mechanism present in the original formulation where the spanwise periodicity of the wave on a periodic mean flow implies a discrete rather than a continuous spectrum of modes. In order to include this aspect in our analysis we now choose the vortex $z$-scale to be large of order $\epsilon^{-1 / 2}$, and define $Z=\epsilon^{1 / 2} z$ as the new independent variable along the span in the pressure equation (1.4). This is linked with the small- $\beta$ limit in $\S 2$. The mean velocity is hence written as

$$
u_{0}=U_{0}(y)+\epsilon^{3} U_{1}\left(\frac{y-y_{c}}{\epsilon}, Z\right),
$$

cf. (2.1). The solution for the wave pressure in the main part of the flow again proceeds in powers of $\epsilon$, the main difference with the previously used form (2.2) being that the leading term in the expansion

$$
\bar{p}=A(Z) \bar{p}_{0}(y)+\epsilon \bar{p}_{1}(y, Z)+\ldots
$$

has an unknown slowly varying amplitude $A(Z)$. This does not affect the main-order eigenvalue formulation (2.3), and we continue to use $\bar{p}_{0}(y)$ for a solution which depends on $y$ only and if necessary can be determined uniquely by assigning a definite value of the pressure somewhere in the flow. For the next term in (3.2), however, we obtain the equation

$$
\left(U_{0}-c_{0}\right)\left(\frac{\partial^{2} \bar{p}_{1}}{\partial y^{2}}-\alpha_{0}^{2} \bar{p}_{1}\right)-2 U_{0}^{\prime} \frac{\partial \bar{p}_{1}}{\partial y}=\left(2 \alpha_{0} \alpha_{1} A-A^{\prime \prime}\right)\left(U_{0}-c_{0}\right) \bar{p}_{0}+c_{1}\left(\bar{p}_{0}^{\prime \prime}-\alpha_{0}^{2} \bar{p}_{0}\right) A,
$$

containing an extra term on the right-hand side compared with (2.4). The inner-layer solution remains virtually unaltered from before except that the amplitude factor $A(Z)$ appears in (2.7) and (2.8) and onwards leading eventually to a condition on the third derivative of $\bar{p}_{1}$ in the form

$$
\frac{\partial^{3} \bar{p}_{1}}{\partial y^{3}}=-A(Z) \frac{2 \alpha_{0}^{2} p_{00} b_{3} c_{1}}{b_{1}^{2}}\left[\ln \left|y-y_{c}\right|+\frac{3 b_{1}}{b_{3} c_{1}} A^{ \pm}(Z)+o(1)\right],
$$

as $y \rightarrow y_{c} \pm 0$, cf. (2.13). The expression for the jump $A^{+}-A^{-}$remains the same as in (2.12). The solvability of equation (3.3) with the requirement (3.4) and the trivial boundary conditions $\bar{p}_{1}^{\prime}(0)=\bar{p}_{1}(\infty)=0$ therefore yields the equation

$$
c_{1} q_{1} A+\left(2 \alpha_{0} \alpha_{1} A-A_{Z Z}\right) q_{2}=A \frac{\alpha_{0}^{2} p_{00}^{2}}{b_{1}^{3}}\left[\int_{-\infty}^{\infty} K_{1}(Y, Z) \frac{\mathrm{d} Y}{Y-c_{1} b_{1}^{-1}}+\frac{\mathrm{i} \pi b_{3} c_{1}}{b_{1}}\right]
$$


for the amplitude function $A(Z)$, using the previous notation for the curvature, $K_{1}=\partial^{2} U_{1} / Y^{2}$. The integral term in (3.5) again indicates the logarithmic singularity at the critical level $Y=c_{1} b_{1}^{-1}$ in the case of neutral disturbances, cf. (2.10) and (2.23).

It is supposed now that the scaled spanwise period of the vortices is $2 \pi / \tilde{\beta}$. The change of variables

$$
\begin{gathered}
\zeta=\tilde{\beta} Z, \quad \tilde{\alpha}=-2 \alpha_{0} \alpha_{1}, \quad \tilde{c}=c_{1} b_{1}^{-1}, \quad s_{1}=-q_{1} b_{1} q_{2}^{-1}, \\
s_{2}=\pi b_{3} s_{3}, \quad s_{3}=\alpha_{0}^{2} p_{00}^{2} q_{2}^{-1} b_{1}^{-3},
\end{gathered}
$$

then leaves equation (3.5) in the form

$$
\tilde{\beta}^{2} A_{\zeta \zeta}+\left[\tilde{\alpha}+\left(s_{1}+\mathrm{i} s_{2}\right) \tilde{c}+s_{3} \int_{-\infty}^{\infty} K_{1}(Y, \zeta) \frac{\mathrm{d} Y}{Y-\tilde{c}}\right] A=0
$$

with the real constants $s_{1-3}$. For neutral disturbances treated as limit solutions with the imaginary part $\tilde{c}_{i} \rightarrow 0+$ the integral above is replaced by

$$
\int_{-\infty}^{\infty} K_{1}(Y, \zeta) \frac{\mathrm{d} Y}{Y-\tilde{c}}=\mathrm{i} \pi K_{1}(\tilde{c}, \zeta)+\mathrm{PV} \int_{-\infty}^{\infty} K_{1}(Y, \zeta) \frac{\mathrm{d} Y}{Y-\tilde{c}}
$$

where PV refers to the principal value of the improper integral.

The usual conclusions concerning periodic, quasi-periodic and decaying/growing (in

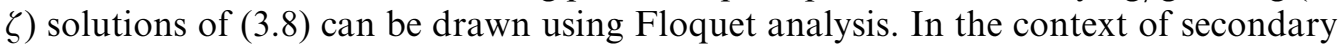
instability, physically sensible distributions of the wave pressure must be bounded as $\zeta \rightarrow \pm \infty$. Narrowing the class of admissible solutions even further, we shall illustrate next the appearance of non-unique unstable and neutral waves with a scaled spanwise period $2 \pi$ (equal to the period of the main-flow vortex) in the limiting cases of small and large $\tilde{\beta}$. This will immediately bring in the issue of modal symmetry also inherent in solutions of (3.8). First, for shorter-scale vortices, $\tilde{\beta} \rightarrow \infty$, the amplitude function and the disturbance parameters expand as

$$
\begin{gathered}
A=A_{n}^{(0)}(\zeta)+\tilde{\beta}^{-2} A_{n}^{(1)}(\zeta)+\ldots, \\
\tilde{\alpha}=\tilde{\beta}^{2} n^{2}+\alpha^{(1)}+\ldots, \quad \tilde{c}=c^{(1)}+\ldots,
\end{gathered}
$$

respectively, where $n=0,1,2, \ldots$ corresponds to the mode number and $A_{n}^{(0)}(\zeta)$ is taken to be either $\cos n \zeta$ or $\sin n \zeta$ (depending on whether the mode is assumed to be even or odd in $\zeta$ ) on account of the main-order balance in (3.8). For the first correction term in (3.10) we then obtain the forced equation

$$
\frac{\mathrm{d}^{2} A_{n}^{(1)}}{\mathrm{d} \zeta^{2}}+n^{2} A_{n}^{(1)}=-A_{n}^{(0)}\left[\alpha^{(1)}+\left(s_{1}+\mathrm{i} s_{2}\right) c^{(1)}+s_{3} \int_{-\infty}^{\infty} K_{1}(Y, \zeta) \frac{\mathrm{d} Y}{Y-c^{(1)}}\right] .
$$

So the requirement of periodicity of $A_{n}^{(1)}$ yields the relation

$$
\int_{0}^{2 \pi}\left[A_{n}^{(0)}(\zeta)\right]^{2}\left[\alpha^{(1)}+\left(s_{1}+\mathrm{i} s_{2}\right) c^{(1)}+s_{3} \int_{-\infty}^{\infty} K_{1}(Y, \zeta) \frac{\mathrm{d} Y}{Y-c^{(1)}}\right] \mathrm{d} \zeta=0,
$$

between the phase speed $c^{(1)}$ and the wavenumber $\alpha^{(1)}$. If in particular the mode number $n=0$ and $A_{0}^{(0)}=1$, then the integral condition (3.13) shows that the relation $c^{(1)}\left(\alpha^{(1)}\right)$ is determined by the $\zeta$-independent vortex component, in agreement with the result (2.22) of the previous section. In contrast, the dispersion relation for higher modes with 
$n>0$ is formed by the average coupled with the $2 n$th harmonic in the Fourier decomposition of the vortex curvature. If, for instance,

$$
K_{1}(Y, \zeta)=\frac{\partial^{2} U_{1}}{\partial Y^{2}}=K_{m}(Y)+K_{\beta}(Y) \cos \zeta+K_{2 n}(Y) \cos 2 n \zeta
$$

cf. (2.14), then for the $n$th neutral mode we have the two relations

$$
\begin{gathered}
s_{2} c^{(1)}+\pi s_{3}\left[K_{m}\left(c^{(1)}\right)+\frac{1}{2} K_{2 n}\left(c^{(1)}\right)\right]=0, \\
\alpha^{(1)}+s_{1} c^{(1)}+s_{3} \mathrm{PV} \int_{-\infty}^{\infty}\left(K_{m}+\frac{1}{2} K_{2 n}\right) \frac{\mathrm{d} Y}{Y-c^{(1)}}=0,
\end{gathered}
$$

from the imaginary and real parts of (3.13) with (3.9). The phase speed $c^{(1)}$, and hence the location of the critical layer in the inner region, are determined by (3.15) and depend on both curvature terms $K_{m}$ and $K_{2 n}$, while the neutral wavenumber follows from (3.16).

Comparing the shorter-scale limit above with the analysis in $\$ 2$ where the planar disturbance, $n=0$, was assumed to be the basic-wave solution we notice that, according to (3.10) and (3.11) the vortex periodicity allows for some freedom in the choice of the main-order oblique wave, although the spectrum is narrowed down to the discrete set by the wave periodicity requirement.

The large $-\tilde{\beta}$ analysis holds also for finite values of $\tilde{\beta}$ and large mode numbers $n$, as inferred from the wavenumber expansion (3.11) and from (3.10) where $A_{n}^{(1)}$ is $O\left(n^{-2}\right.$ $A_{n}^{(0)}$ ) at large $n$ on account of (3.12). On the other hand, if the mode number is large but $\tilde{\beta} \rightarrow 0$, the asymptotic expansions (3.10) and (3.11) fail when $n \tilde{\beta}$ is $O(1)$.

Further analysis is also possible when $\tilde{\beta} \rightarrow 0$ for a fixed $n$ or, more generally, if $\tilde{\beta} n \ll 1$. For the curvature function in the form (3.14) with $K_{2 n}=0$, for instance, the problem reduces to the conventional Mathieu equation (see e.g. Abramowitz \& Stegun 1970 , and references therein). The limit periodic solutions with $\tilde{\beta}^{2} \ll 1$ then tend to become concentrated near the extremum points of $\cos \zeta$, as implied by a WKB argument in the main part of the period and a local reduction to the Weber equation for the core of the solution near the locations $\zeta=\zeta_{0}+2 n \pi$, with integer $n$ and $0 \leqslant \zeta_{0}<2 \pi$; there the expression in square brackets in (3.8) denoted as $F(\tilde{c}, \tilde{\alpha}, \zeta)$ satisfies the stationary-point conditions

$$
F(\tilde{c}, \tilde{\alpha}, \zeta)=0, \quad F_{\zeta}(\tilde{c}, \tilde{\alpha}, \zeta)=0 .
$$

In application to the Mathieu equation, the second requirement above yields sin $\zeta_{0}=0$, to fix the position of the core region at $\zeta_{0}=0$ or $\zeta_{0}=\pi$, for the two independent sets of limit solutions, and then the first requirement provides the main-order dispersion relation between $\tilde{c}$ and $\tilde{\alpha}$.

For a more general vortex, however, and especially in the case of neutral waves, the two complex equations (3.17) lead to an overspecified formulation for the three real quantities $\tilde{c}, \tilde{\alpha}, \zeta_{0}$, which tends to indicate that the stationary points of the limit solutions are shifted from the real axis into the complex $\zeta$-plane; for a similar feature in a non-periodic problem see e.g. Stewartson \& Leibovich (1987). Below we demonstrate the derivation of the neutral-wave characteristics for the case of a small shift comparable initially with the typical size of the core region. Suppose that the vortex curvature and the wave parameters can be expanded in the form

$$
\begin{aligned}
& K_{1}(Y, \zeta)=K^{(0)}(Y, \zeta)+\tilde{\beta}^{1 / 2} \tilde{\delta} K^{(1)}(Y, \zeta)+\ldots, \\
& \{\tilde{c}, \tilde{\alpha}\}=\left\{\tilde{c}_{0}, \tilde{\alpha}_{0}\right\}+\tilde{\beta}^{1 / 2}\left\{\tilde{c}_{1}, \tilde{\alpha}_{1}\right\}+\tilde{\beta}\left\{\tilde{c}_{2}, \tilde{\alpha}_{2}\right\}+\ldots,
\end{aligned}
$$


where the curvature term $K^{(0)}$ corresponds to the stationary point on the real axis at $\zeta=\zeta_{0}$, and the $O(1)$ parameter $\tilde{\delta}$ characterizes a shift from $\zeta_{0}$. The conditions (3.17) hold in the first two approximations, $O(1)$ and $O\left(\tilde{\beta}^{1 / 2}\right)$, so that

$$
\begin{gathered}
\tilde{\alpha}_{0}+\left(s_{1}+\mathrm{i} s_{2}\right) \tilde{c}_{0}+s_{3}\left[\mathrm{i} \pi K_{0}^{(0)}\left(\tilde{c}_{0}, \zeta_{0}\right)+\mathrm{PV} \int_{-\infty}^{\infty} K_{0}^{(0)}\left(Y, \zeta_{0}\right) \frac{\mathrm{d} Y}{Y-\tilde{c}_{0}}\right]=0 \\
\tilde{\alpha}_{1}+\left(s_{1}+\mathrm{i} s_{2}\right) \tilde{c}_{1}+s_{3} \mathrm{i} \pi\left[\tilde{c}_{1} \frac{\partial K_{0}^{(0)}\left(\tilde{c}_{0}, \zeta_{0}\right)}{\partial Y}+\tilde{\delta} K_{0}^{(1)}\left(\tilde{c}_{0}, \zeta_{0}\right)\right] \\
+\tilde{c}_{1} s_{3} \frac{\partial}{\partial \tilde{c}_{0}}\left[\mathrm{PV} \int_{-\infty}^{\infty} K_{0}^{(0)}\left(Y, \zeta_{0}\right) \frac{\mathrm{d} Y}{Y-\tilde{c}_{0}}\right]+\tilde{\delta} s_{3} \mathrm{PV} \int_{-\infty}^{\infty} K_{0}^{(1)}\left(Y, \zeta_{0}\right) \frac{\mathrm{d} Y}{Y-\tilde{c}_{0}}=0,
\end{gathered}
$$

where we have assumed the local behaviour

$$
\begin{gathered}
K^{(0)}=K_{0}^{(0)}\left(Y, \zeta_{0}\right)+\frac{1}{2}\left(\zeta-\zeta_{0}\right)^{2} K_{2}^{(0)}\left(Y, \zeta_{0}\right)+\ldots, \\
K^{(1)}=K_{0}^{(1)}\left(Y, \zeta_{0}\right)+\left(\zeta-\zeta_{0}\right) K_{1}^{(1)}\left(Y, \zeta_{0}\right)+\ldots,
\end{gathered}
$$

in the neighbourhood of $\zeta_{0}$. The equations (3.20) and (3.21) serve to determine $\tilde{\alpha}_{0}, \tilde{c}_{0}, \tilde{\alpha}_{1}, \tilde{c}_{1}$. In the core region, where $\zeta-\zeta_{0}=\tilde{\beta}^{1 / 2} \theta$ with $\theta$ of $O(1)$, the amplitude equation (3.8) then acquires the form

where

$$
A_{\theta \theta}+\left(\Lambda_{1}+\Lambda_{2} \theta+\Lambda_{3} \theta^{2}\right) A=0,
$$

$$
\begin{gathered}
\Lambda_{1}=\tilde{c}_{2}+\left(s_{1}+\mathrm{i} s_{2}\right) \tilde{c}_{2}+s_{3} \mathrm{i} \pi\left[\tilde{c}_{2} \frac{\partial K_{0}^{(0)}\left(\tilde{c}_{0}, \zeta_{0}\right)}{\partial \tilde{c}_{0}}+\frac{1}{2} \tilde{c}_{1}^{2} \frac{\partial^{2} K_{0}^{(0)}\left(\tilde{c}_{0}, \zeta_{0}\right)}{\partial \tilde{c}_{0}^{2}}+\tilde{\delta} \tilde{c}_{1} \frac{\partial K_{0}^{(1)}\left(\tilde{c}_{0}, \zeta_{0}\right)}{\partial \tilde{c}_{0}}\right] \\
+s_{3} \frac{\tilde{c}_{1}^{2}}{2} \frac{\partial^{2}}{\partial \tilde{c}_{0}^{2}}\left(\mathrm{PV} \int_{-\infty}^{\infty} K_{0}^{(0)}\left(Y, \zeta_{0}\right) \frac{\mathrm{d} Y}{Y-\tilde{c}_{0}}\right)+s_{3} \tilde{c}_{2} \frac{\partial}{\partial \tilde{c}_{0}}\left(\mathrm{PV} \int_{-\infty}^{\infty} K_{0}^{(0)}\left(Y, \zeta_{0}\right) \frac{\mathrm{d} Y}{Y-\tilde{c}_{0}}\right) \\
+\tilde{\delta}_{3} \tilde{c}_{1} \frac{\partial}{\partial \tilde{c}_{0}}\left(\mathrm{PV} \int_{-\infty}^{\infty} K_{0}^{(1)}\left(Y, \zeta_{0}\right) \frac{\mathrm{d} Y}{Y-\tilde{c}_{0}}\right) \\
\Lambda_{2}=\tilde{\delta s_{3}}\left[\mathrm{i} \pi K_{1}^{(1)}\left(\tilde{c}_{0}, \zeta_{0}\right)+\mathrm{PV} \int_{-\infty}^{\infty} K_{1}^{(1)}\left(Y, \zeta_{0}\right) \frac{\mathrm{d} Y}{Y-\tilde{c}_{0}}\right] \\
\Lambda_{3}=\frac{1}{2} s_{3}\left[\mathrm{i} \pi K_{2}^{(0)}\left(\tilde{c}_{0}, \zeta_{0}\right)+\mathrm{PV} \int_{-\infty}^{\infty} K_{2}^{(0)}\left(Y, \zeta_{0}\right) \frac{\mathrm{d} Y}{Y-\tilde{c}_{0}}\right]
\end{gathered}
$$

Solutions of the Weber equation (3.24) satisfying the decay conditions, $A \rightarrow 0$ as $\theta \rightarrow \pm \infty$, exist, provided that

$$
\left(-\frac{1}{4 \Lambda_{3}}\right)^{1 / 2}\left(\Lambda_{1}-\frac{\Lambda_{2}^{2}}{4 \Lambda_{3}}\right)=-n-\frac{1}{2}
$$

with integer $n=0,1, \ldots$, and $\arg \left[\left(-1 / \Lambda_{3}\right)^{1 / 2}\right]<\frac{1}{2} \pi$ (see e.g. Abramowitz \& Stegun 1970). For $\Lambda_{2}=0$, or after a suitable shift in $\theta$ otherwise, the wave solutions determined by (3.24) with (3.28) may be again divided into families of odd and even functions (cf. HH). So, the values of $\tilde{\alpha}_{2}, \tilde{c}_{2}$ are fixed by (3.28), using (3.25)-(3.27). Also according to (3.24) for large values of $\tilde{\delta}$ in (3.18) the core part of the local solution is shifted further away from the real axis into the vicinity of the point $\zeta_{0}-\frac{1}{2} \tilde{\beta}^{1 / 2} \Lambda_{2} \Lambda_{3}^{-1}$, with the imaginary part of the shift being proportional to $\tilde{\delta}$, on account of (3.26) and (3.27). Asymptotic solutions of (3.8) with a finite distance between the stationary point and the real $\zeta$-axis exhibit stronger dependence on analytic properties of the curvature function in the complex plane $\zeta$. 


\section{Specific examples}

Here, we illustrate solutions of (3.8) and (3.9) for three specific examples of model vortex flows. Our main concern will be with the effect of spanwise period on the wave characteristics, and with possible deviations from the short-/long-scale limit properties described in $\S 3$. The vortex distributions below are symmetric in $\zeta$, and both even and odd wave modes were found to be possible; however, for brevity the results are presented for the even waves only.

For our first example we take a smooth curvature distribution, namely

$$
K_{1}(Y, \zeta)=(\mu+\nu \cos \zeta)\left(1+Y^{2}\right)^{-1}
$$

with constants $\mu, \nu$. Solutions of (3.8) with the periodicity conditions $A(0)=A(2 \pi)$, $A^{\prime}(0)=A^{\prime}(2 \pi)$ were obtained numerically using a second-order accurate finitedifference approximation of the equation with the explicit normalization $A(0)=1$. Properties of the first four neutral-wave solutions with the mode numbers $n=0-3$ are illustrated in figures 2 and 3 . The limit behaviour of large- $\tilde{\beta}$ waves is governed by (3.11), whereas for $\tilde{\beta} \rightarrow 0$ the core of the amplitude is centred around one of the two stationary points, in our case at $\zeta=0$, (the limit values $\tilde{\alpha}=-0.2167, \tilde{c}=0.6823$ ), or at $\zeta=\pi(\alpha=0.2589, \tilde{c}=1.2134$ in the limit) as follows from (3.17) with (4.1). The most peculiar feature here is the continuation of the third mode, $n=2$ in figure 2 , towards the small- $\tilde{\beta}$ limit with the larger phase speed, with the core near $\zeta=\pi$, as opposed to the limit for the modes $0,1,3$ centred at $\zeta=0,2 \pi$ (see also figure 3 ). This is obviously a consequence of the complex coefficients in the Mathieu equation (3.8) (cf. the real case in e.g. Abramowitz \& Stegun 1970). It was verified that modes 1 and 2 have no common points, although the paths of the two roots in $(\tilde{\alpha}, \tilde{c}, \tilde{\beta})$ space become very close when $\tilde{\beta} \approx 0.4-0.5$. The approach of the large- $\tilde{\beta}$ solutions in figure 3 to simple harmonics, the appearance of concentrated amplitudes at smaller $\tilde{\beta}$, and a fairly accurate reproduction of the limit values at $\tilde{\beta}=0$ in figure 2 , all this is in favourable agreement with the conclusions drawn in $\S 3$ for a general smooth vortex field.

In the second example the curvature remains smooth across the boundary layer with, however, piecewise continuity along the span according to the formulae

$$
\begin{aligned}
K_{1}(Y, \zeta) & =\Phi(\tilde{\zeta})\left(1+Y^{2}\right)^{-1} \quad \text { where } \tilde{\zeta}=\zeta \beta^{-1} \\
\Phi(\tilde{\zeta}) & =\Phi_{+} \quad \text { if } 0 \leqslant \tilde{\zeta}<\tilde{\zeta}_{0} \text { or } \tilde{\zeta}_{0}+\tilde{\zeta}_{1}<\tilde{\zeta} \leqslant \tilde{\zeta}_{1}+2 \tilde{\zeta}_{0} \\
\Phi(\tilde{\zeta}) & =-\Phi_{-} \text {if } \tilde{\zeta}_{0} \leqslant \tilde{\zeta} \leqslant \tilde{\zeta}_{0}+\tilde{\zeta}_{1}
\end{aligned}
$$

over the first period $0 \leqslant \tilde{\zeta} \leqslant \tilde{\zeta}_{1}+2 \tilde{\zeta}_{0}$, with constant parameters $\tilde{\zeta}_{0}, \tilde{\zeta}_{1}, \Phi_{ \pm}$. The problem can be solved analytically for both neutral and growing disturbances, and the resulting dispersion relation allows then a good deal of analysis. For example, the neutral wavenumber $\tilde{\alpha}$ against the length parameter $\tilde{\zeta}_{1}$ for the first six even modes in the case $\Phi_{+}=s_{1-3}=1,2 \tilde{\zeta}_{0}=\tilde{\zeta}_{1}$ and variable period-averaged vorticity is shown in figure 4 . As the quantity $\Phi_{+}-\Phi_{-}$decreases from 1 to 0 the well-behaved pattern of the neutral curves in figure 4( $a$ ) becomes non-monotonic for the modes $n=1,3,5$ (figure $4 b$ ) and eventually splits into almost disconnected loops formed by the branches with odd and even numbers (figure 4c). The high flow symmetry in the last illustration brings in rather interesting wave properties. The phase speed turns out to be zero on branches $0-5$. Along the branches marked as $0^{\prime}-5^{\prime}$ the phase speed is non-zero in general, although for each pair of the intersecting loops there exists a common point with equal $\tilde{\alpha}$ and $\tilde{c}=0$ (dots in figure $4 c$ ). If we add the third coordinate $\tilde{c}_{i}$ to the axes $\tilde{\zeta}_{1}, \tilde{\alpha}$ then the solutions can be presented as an infinite sequence of surfaces which cross the plane $\tilde{c}_{i}=0$ along the neutral curves. The mode coupling then turns out to be structurally unstable to small changes in the growth rates, as illustrated in figure 5 which also 


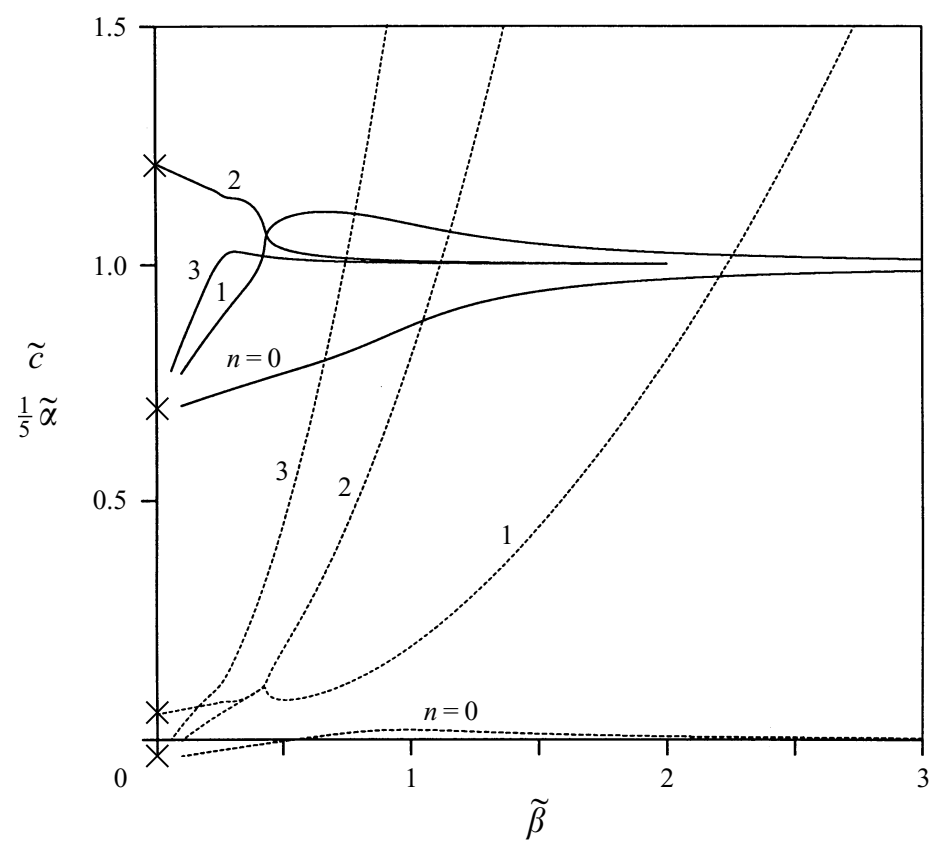

FIGURE 2. Neutral parameters plotted against the spanwise wavenumber $\tilde{\beta}$, from numerical solution of (3.8), (4.1) with $s_{1}=s_{3}=1, s_{2}=-1, \mu=2 / \pi, v=-1 / \pi$, for the first four even modes of wave disturbances; - , the phase speed $\tilde{c} ;---$, the wavenumber $\frac{1}{5} \tilde{\alpha}$; the values $n=0-3$ refer to the mode number. Crosses correspond to the stationary-point limit values $\tilde{c}=0.6823, \tilde{\alpha}=-0.2167$ and $\tilde{c}=1.2134, \tilde{\alpha}=0.2589$.

suggests a saddle point in the shape of the instability surfaces in the $\tilde{\zeta}_{1}, \tilde{\alpha}, \tilde{c}_{i}$ space near the common points.

In our third example the vortex curvature has discontinuities along the span as before and, in addition, jumps across the layer, specifically we define

$$
K_{1}=\Phi(\tilde{\zeta}) \quad \text { if } \quad Y_{0} \leqslant Y \leqslant Y_{1} ; \quad K_{1} \equiv 0 \quad \text { if } \quad Y>Y_{1} \quad \text { or } \quad Y<Y_{0}
$$

where $Y_{0,1}$ are constant and $\Phi(\tilde{\zeta})$ is given in (4.2). This model has certain (although fairly loose) similarities with the concentrated nonlinear Görtler vortices of Hall \& Lakin (1988). The dispersion relation is again derivable analytically for even and odd, stable and unstable disturbances. The neutral wavenumbers for the first several even modes are shown in figure 6(a). The disturbance phase speed is now found to be bounded in the range $Y_{0}<\tilde{c}<Y_{1}$ (see figure $6 b$ ). The short-wave branches in figure $6(a)$ originating at a finite-limit wavenumber for the first mode and going upwards to infinity for the higher modes are qualitatively similar to those in the previous examples (cf. figures 2 and 4). The most striking property of the current model, however, is the reversal of the neutral curves into the region of shorter spanwise scales after reaching a certain threshold, with a clear trend to an unbounded wavenumber growth as the flow period decreases. The plots of the neutral curves in the plane $\tilde{c}, \tilde{\alpha}$ in figure $6(b)$ indicate a fast approach of the phase speed to one of the two limit values $\tilde{c}= \pm 2$, with the location of the critical layer being shifted towards the vortex boundaries at $Y=Y_{0,1}= \pm 2$. Hence the reversal of the plots in figure 6(a), and consequent nonexistence of larger-scale neutral modes, is due to the logarithmic singularities in the integral term in the equation (3.8).

Further computational and analytical details on all three examples considered briefly in this section are available from the authors or the Journal Editorial Office. 
(a)

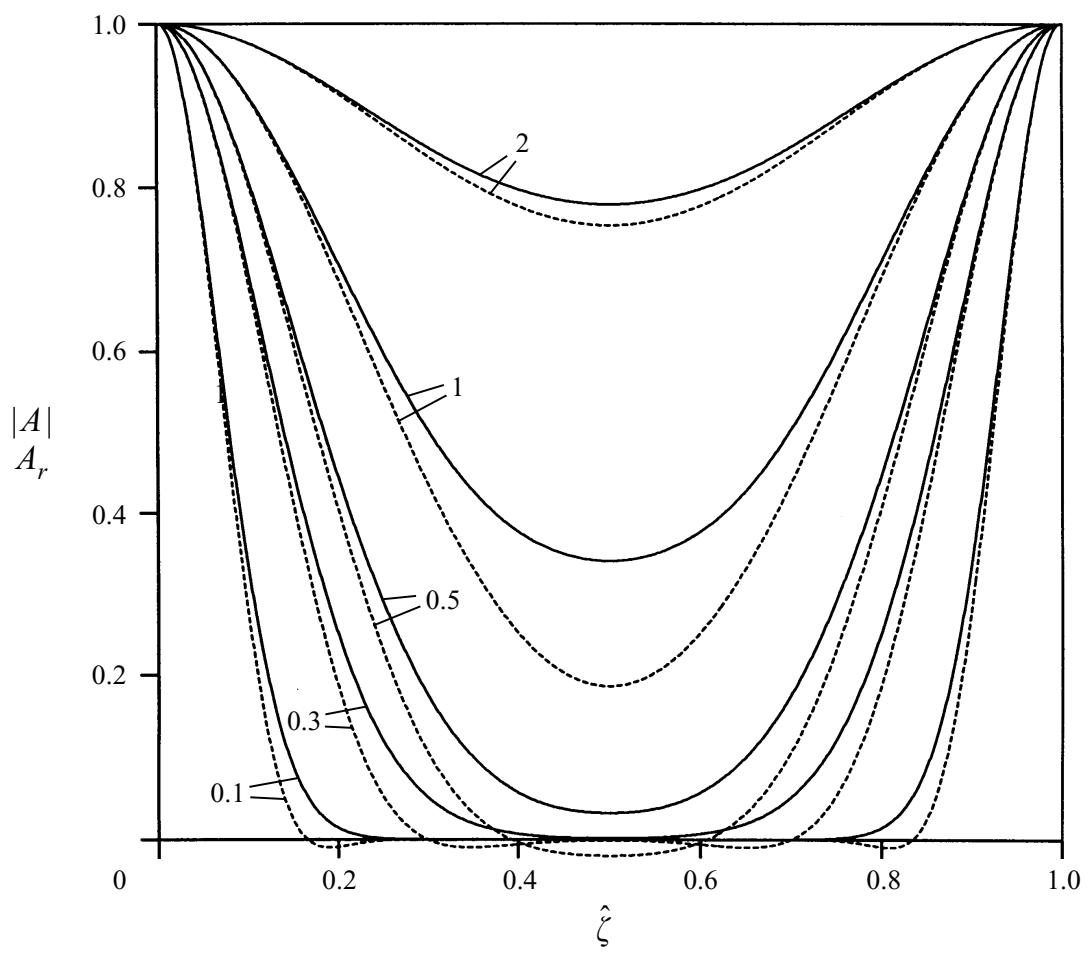

(b)

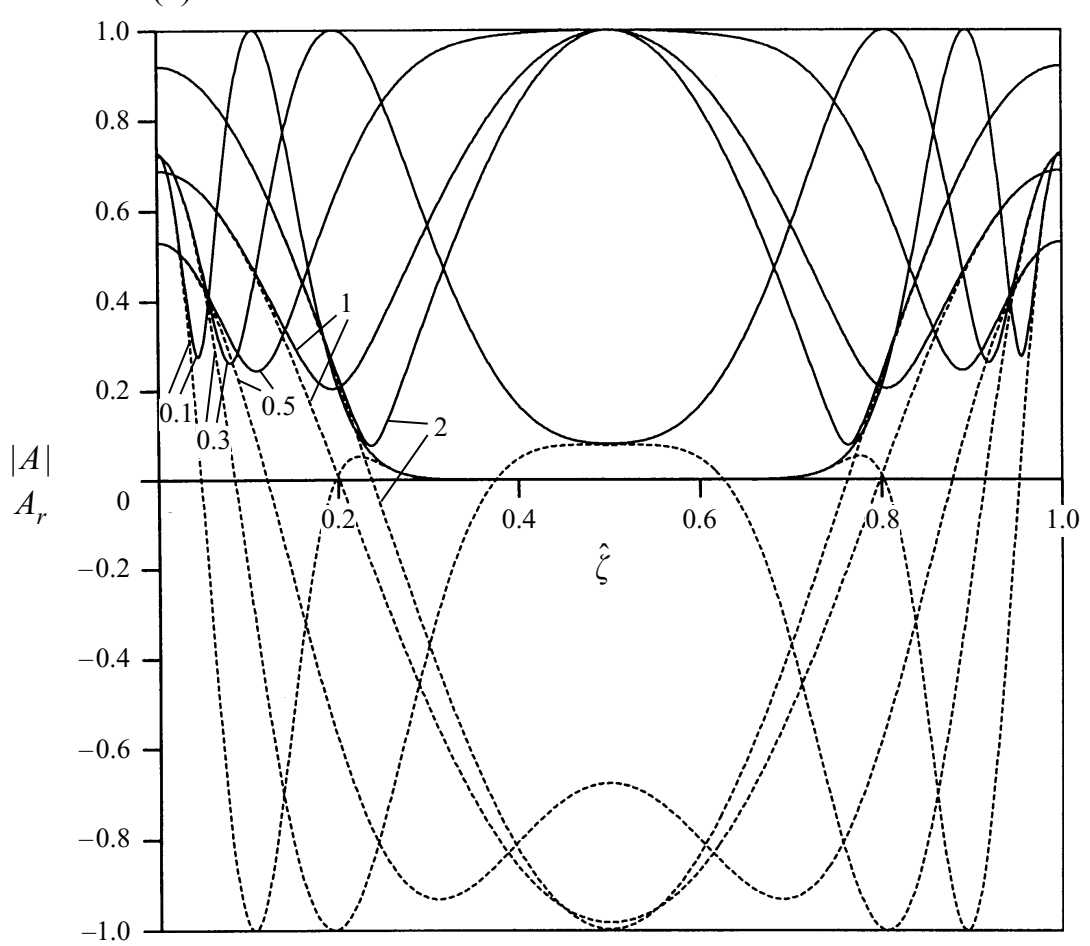

FigURE 3. For caption see p. 157. 

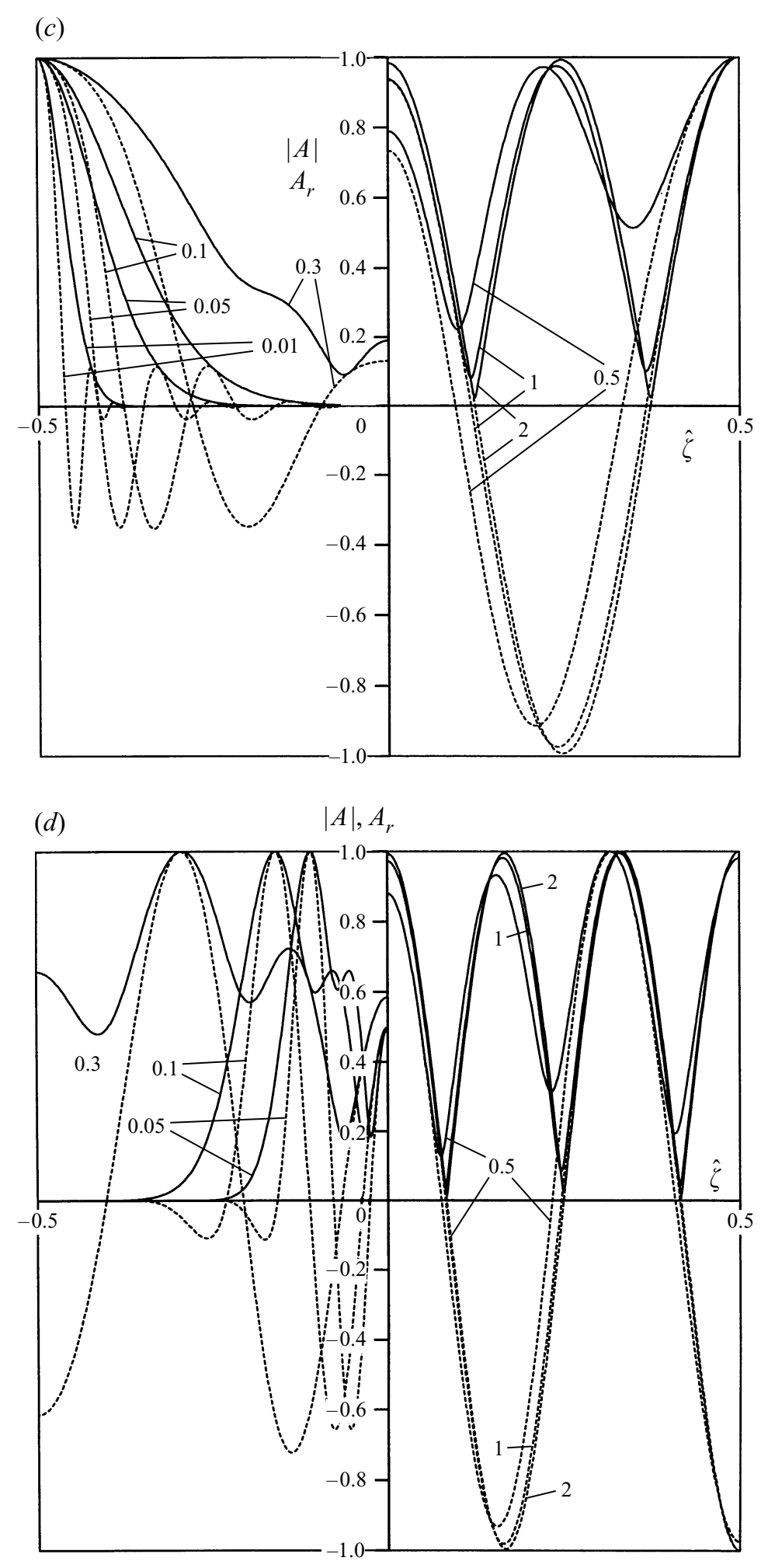

FIGURE 3. For caption see facing page. 
(a)

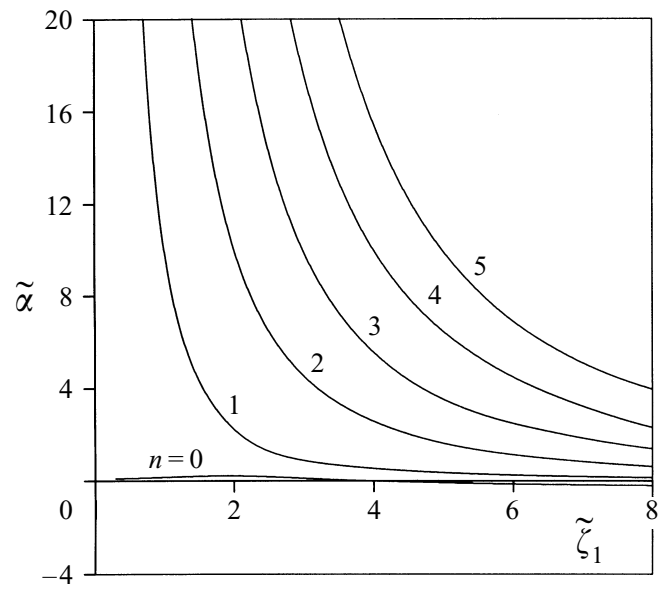

(b)

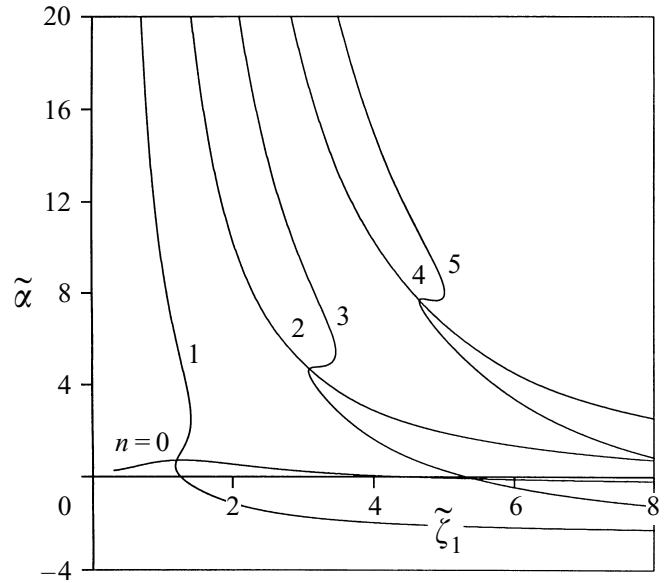

(c)

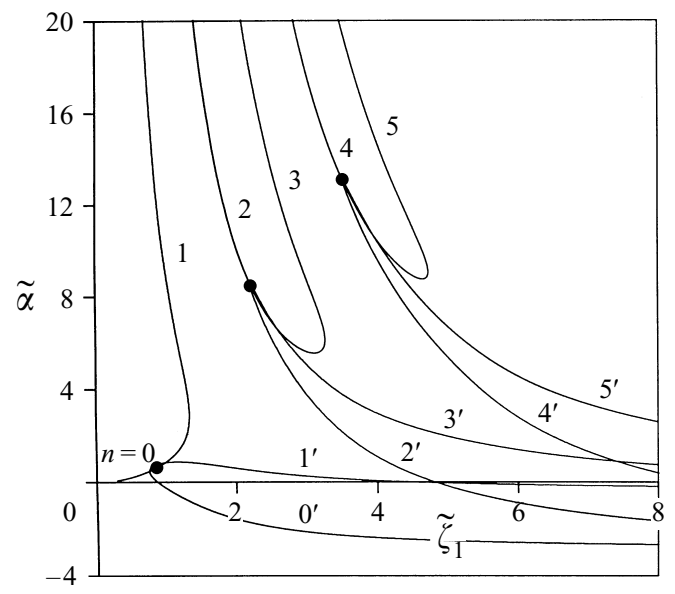

FIGURE 4 . The neutral wavenumber $\tilde{\alpha}$ plotted against the spanwise length parameter $\tilde{\zeta}_{1}$ (= half-length of the period) for the second model flow, with the curvature (4.2); even modes with $s_{1-3}=\Phi_{+}=1$ and $(a) \Phi_{-}=0 ;(b) \Phi_{-}=0.8 ;(c) \Phi_{-}=1$; the mode numbers $n=0-5$ are shown in the figures. The mode splitting in $(c)$ results in reconnections of the branches 0 and $1,0^{\prime}$ and $1^{\prime}$, etc., with the dots indicating the location of the mode intersections.

\section{Discussion}

We conclude with the following brief remarks concerning the two central applications of the wave pressure equation (1.4) brought out at the beginning of this paper.

In order to apply our analysis to the nonlinear flow configuration examined in Brown et al. (1993) we interpret $\epsilon$ in (2.1) as $\left(X-X_{n}\right)^{1 / 2}$; cf. (1.6). The actual intensity of the starting vortex is $\epsilon$ times weaker than in (2.1), of order $\epsilon^{4}$, but even this

FIGURE 3. - The absolute value and ---, the real part of the amplitude function $A$ plotted against the scaled spanwise variable $\hat{\zeta}=\zeta /(2 \pi)$ for various $\hat{\beta}$, from computations for figure 2 . The solution functions are scaled to have a unit maximum modulus over the period; $(a)-(d)$ correspond to mode numbers $0-3$, respectively; the values of $\tilde{\beta}$ are indicated at the plots; $(a, b)$ the amplitude function shown for the first period; $(c, d)$ plots for small and large $\tilde{\beta}$ are shown on separate half-periods. 


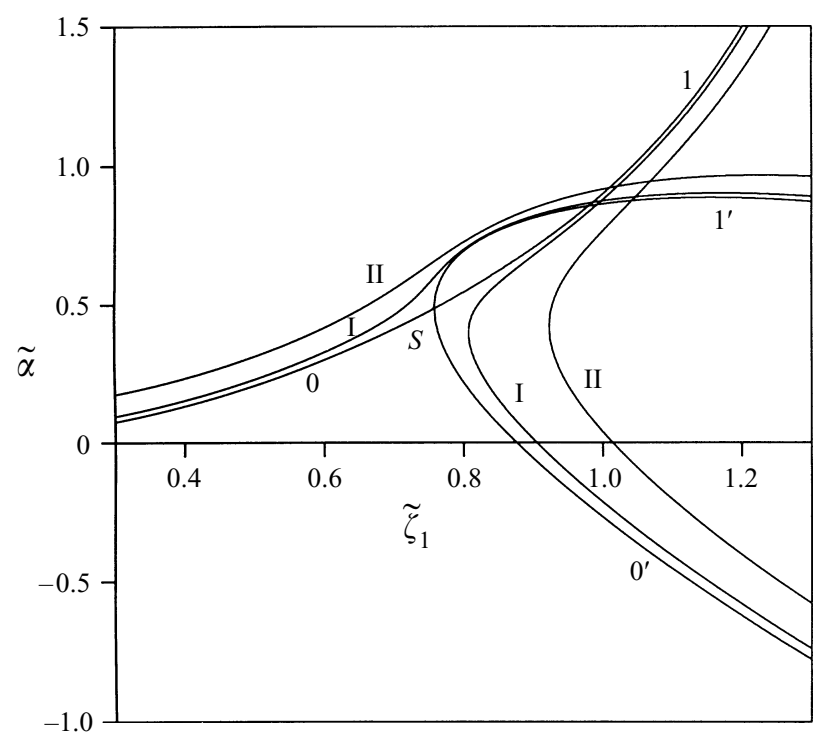

FIGURE 5. Contours of constant growth rate in the plane $\tilde{\zeta}_{1}, \tilde{\alpha}$ near the neutral mode intersection point $S$; model flow (4.2) with $s_{1-3}=\Phi_{+}=1 ; 0,1,0^{\prime}, 1^{\prime}$, the neutral waves; I, growth rate $\tilde{c}_{i}=0.01 ; I I$, $\tilde{c}_{i}=0.05$.

(a)

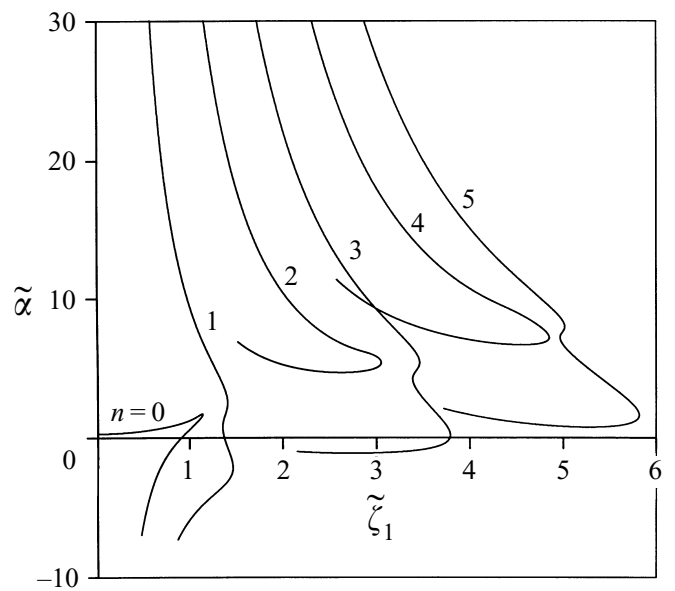

(b)

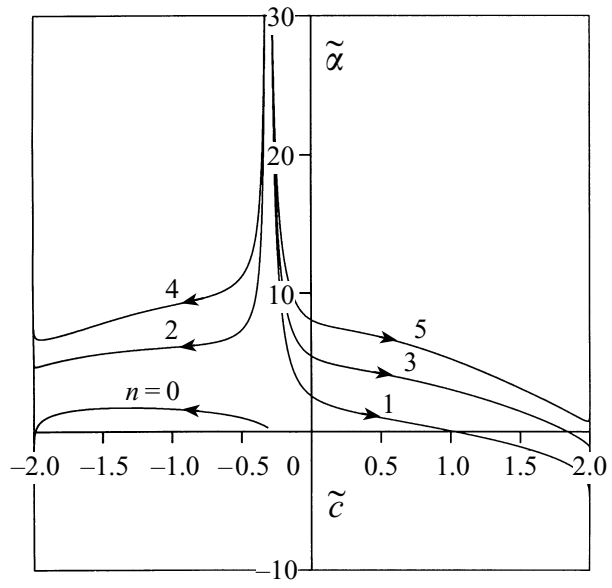

FIGURE 6. (a) The neutral wavenumber for the curvature distribution (4.3) plotted against the length parameter $\tilde{\zeta}_{1}$ for the case $2 \tilde{\zeta}_{0}=\tilde{\zeta}_{1}, s_{1-3}=\Phi_{+}=1, \Phi_{-}=0.8, Y_{0}=-2, Y_{1}=2$, the mode numbers are indicated at the plots; $(b)$ the neutral-wave phase speed against the wavenumber with varying $\tilde{\zeta}_{1}$; arrows indicate the approach to $\tilde{\zeta}_{1}=0$ along the lower branches in $(a)$.

contribution proves to have no effect on the critical-layer singularity because the vortex curvature at $y=y_{c}$ is zero. It is only in the next approximation, of $O\left(\epsilon^{5}\right)$ in (1.6), that the curvature effects enter the reckoning and lead eventually to the constraint (1.7). Owing to the relative weakness of the mechanism involved, the formation of the singularity went unnoticed in the main-order analysis in Smith et al. (1993) of a nonequilibrium interaction preceding the strongly nonlinear saturated stage where a match with the leading approximation in Brown et al. (1993) was found possible.

It should be emphasized that no special adjustments seem to be necessary in the HS- 
theory except that the interactive neutral modes there must be allowed a logarithmic singularity at the critical level. Having relaxed the critical-layer restrictions for the wave, then, the extra conditions on the starting profile found in Brown et al. (1993) become unnecessary, hence leading to a self-consistent description throughout.

The aspect of linear secondary instabilities in vortex flows will most certainly require further computational work for the full equation (1.4) tied up with computations for a more realistic vortex velocity field itself, as also noted independently by Goldstein \& Wundrow (1995). Our theory in this part can provide a useful verification of the numerical technique since no exact solutions of the stability problem to be used for this purpose seem to be available at present. The complexity of the problem can also be illustrated with specific numerical examples of solutions of (3.8) which are only briefly described in $\S 4$. We found that, even with a reasonably simple choice for the vortex distribution, the solution, in addition to the 'natural' multi-mode non-uniqueness in equations with periodic coefficients, can have hysteresis and mode-crossing nonuniqueness in certain cases. The variety of phenomena in stronger vortex flows is likely to be even wider, and here the perturbation analysis again provides useful leads, e.g. to waves with the spanwise period twice as large as the vortex period, to quasi-periodic instabilities, and further still to weakly/strongly nonlinear regimes and to the receptivity issue which takes us back to the study of the full spectrum of instabilities present in the flow.

Further aspects of the theory and applications left out of the scope of this paper include spatial instabilities where a similar treatment can be used, and perturbation approaches based on different basic-wave solutions. For example, a modification of the multi-mode formulation of $\S 3$ to the case of the Goldstein \& Wundrow (1995) nearwall vortices may be useful. Extensions of the theory to axisymmetric configurations, compressible flows (for which singular neutral modes are known to exist even in the two-dimensional approximation), and stationary vortices rather than wave instabilities should also prove of much theoretical and practical interest.

The authors are grateful to Professor S. N. Brown and Dr J. W. Elliott for a number of helpful discussions, to Dr D. W. Wundrow for kindly sending us a copy of his paper with Dr M. E. Goldstein, to referees for pointing out that paper and their 1994 NASA TM report, and to Dr S. R. Otto for kindly noting several other references.

\section{REFERENCES}

Abramowitz, M. \& Stegun, I. A. 1970 Handbook of Mathematical Functions. Dover.

Betchov, R. \& Criminale, W. O. 1967 Stability of Parallel Flows. Academic.

Balachandar, S., Streett, C. L. \& Malik, M. R. 1992 Secondary instability in rotating-disk flow. J. Fluid Mech. 242, 323-347.

Blackaby, N. D. \& Hall, P. 1995 The nonlinear evolution of the inviscid secondary instability of streamwise vortex structures. Phil. Trans. R. Soc. Lond. A 352, 483-502.

Brown, P. G., Brown, S. N., Smith, F. T. \& Timoshin, S. N. 1993 On the starting process of strongly nonlinear vortex/Rayleigh-wave interactions. Mathematika 40, 7-29.

Goldstein, M. E. \& Wundrow, D. W. 1995 Interaction of oblique instability waves with weak streamwise vortices. J. Fluid Mech. 284, 377-407.

Hall, P. 1993 Streamwise vortices in heated boundary layers. J. Fluid Mech. 252, 301-324.

Hall, P. \& Horseman, N. J. 1991 The linear inviscid secondary instability of longitudinal vortex structures in boundary layers. J. Fluid Mech. 232, 357-375 (referred to herein as HH).

Hall, P. \& Lakin, W. D. 1988 The fully nonlinear development of Görtler vortices in growing boundary layers. Proc. R. Soc. Lond. A 415, 421-444. 
Hall, P. \& Smith, F. T. 1991 On strongly nonlinear vortex/wave interactions in boundary-layer transition. J. Fluid Mech. 227, 641-666 (referred to herein as HS).

Henningson, D. S. 1987 Stability of parallel inviscid shear flow with mean spanwise variation. The Aeronautical Research Institute of Sweden, Aerodynamics Department, FFA TN 1987-57.

Hocking, L. M. 1968 Long wavelength disturbances to non-planar parallel flow. J. Fluid Mech. 31, 625-634.

Lin, C. C. 1955 The Theory of Hydrodynamic Stability. Cambridge University Press.

Malik, M. R., LI, F. \& Chang, C.-L. 1994 Crossflow disturbances in three-dimensional boundary layers: nonlinear development, wave interaction and secondary instability. J. Fluid Mech. 268, $1-36$.

Отто, S. R. 1996 On the secondary instability of Görtler vortices in three-dimensional boundary layers. In IUTAM Symposium on Nonlinear Instability and Transition in 3D Boundary Layers, pp. 85-94. Kluwer.

RaYleigh, J. W. S. 1880 On the stability, or instability, of certain fluid motions. Proc. Lond. Math. Soc. 9, 57-70.

Smith, F. T., Brown, S. N. \& Brown, P. G. 1993 Initiation of three-dimensional transition paths from an inflectional profile. Eur. J. Mech. B Fluids 12, 447-473.

Stewartson, K. \& Leibovich, S. 1987 On the stability of a columnar vortex to disturbances with large azimuthal wavenumber: the lower neutral points. J. Fluid Mech. 178, 549-566.

WundRow, D. W. \& Goldstern, M. E. 1994 Nonlinear instability of a unidirectional transversely sheared mean flow. NASA TM 106779. 